\title{
Supplementary Information: Stable
}

\section{two-dimensional materials for oxygen reduction and oxygen evolution reactions}

\author{
Ankit Jain, ${ }^{*},+\ddagger$ Zhenbin Wang, ${ }^{\dagger, \ddagger}$ and Jens K Nørskov ${ }^{*, \dagger}$ \\ $\dagger$ Department of Physics, Technical University of Denmark, Denmark \\ $\ddagger$ Contributed equally to this work \\ E-mail: ankitj@alumni.cmu.edu; jkno@dtu.dk
}

Aqueous Stability Assessing the aqueous stability of materials requires the description of solvated ions. These energies are challenging to obtain from standard DFT calculations. ${ }^{1}$ Alternative approaches utilizing experimentally measured solvation energies by correcting the DFT computed energies of solids have been suggested to overcome this challenge. ${ }^{2,3}$ In this work, a similar approach utilizing experimental solvation energies and a correction scheme for computed DFT energies is employed. ${ }^{3}$ In particular, the methodology for calculating references for solid elements, oxygen gas, solid oxide compounds, water, and hydrogen and other gases is adopted from Ref. ${ }^{3}$ For solvated species, we used raw experimentally measured solvation energies. The correction of these experimentally measured solvation energies to match the formation energies of oxides in the Ref. ${ }^{3}$ approach is, however, avoided in this work. Further details on these calculations can be found in Ref. ${ }^{3}$ and Ref. ${ }^{4}$

The aqueous stability of two-dimensional materials under ORR and OER operating conditions is summarized in Fig. S1(a) and S1(b).

The DFT predicted aqueous stability of compounds were benchmarked by Singh et al. ${ }^{4}$ 


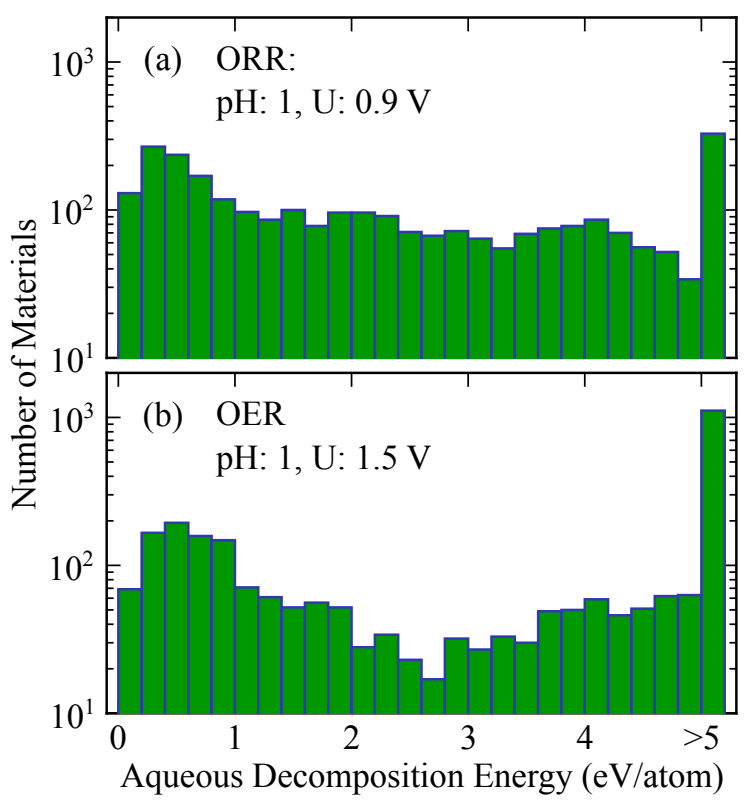

Figure S1: The aqueous stability of materials under (a) ORR and (b) OER operating conditions. Only materials with convex hull energy lower than $0.15 \mathrm{eV} /$ atom are included. All materials from Materials Project are included in the determination of stabilities.

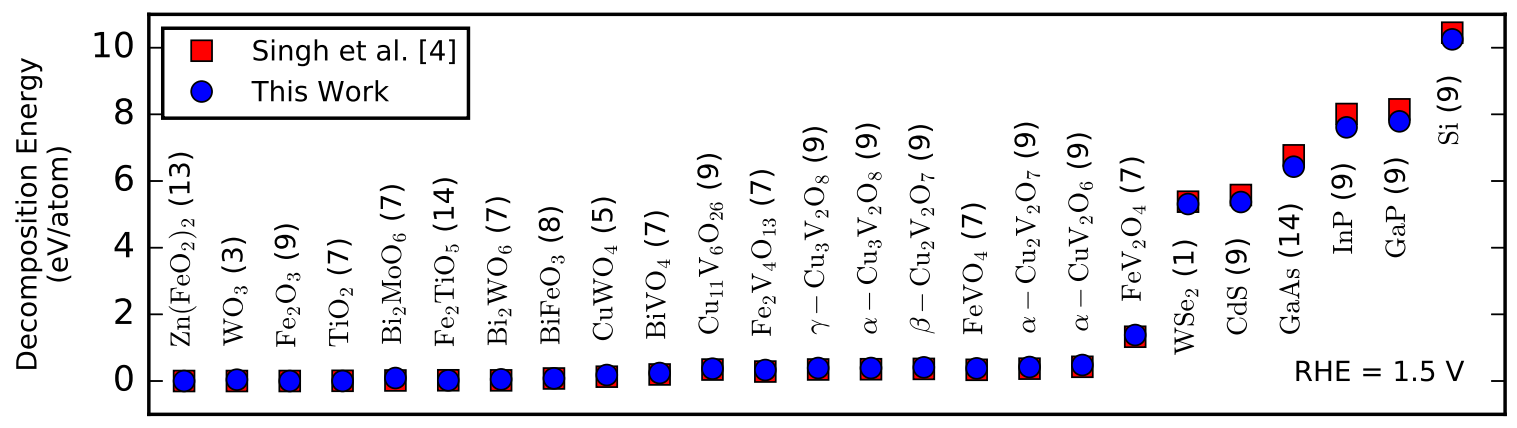

Figure S2: The predicted aqueous decomposition energies of 20 compounds used in $\operatorname{Ref}^{4}$ for comparison against experiments. The decompsoition energies from $\operatorname{Ref}^{4}$ are renormalized here by total number of atoms for comparison. Materials with predicted decomposition energies of up to 0.08 and $0.20 \mathrm{eV} /$ atom are stable in experiments when aqueous species and solid-only decomposition products are involved. 
against the experimentally observed stabilities of 20 compounds. Using the same set of 20 compounds, we find that materials with predicted aqueous decomposition energies of up to $0.20 \mathrm{eV} /$ atom are stable/metastable in experiments, illustrated in Fig.-S1. ${ }^{5}$ This stability threshold of $0.20 \mathrm{eV} /$ atom is higher than the uncertainty of DFT predicted energies compared to experiments ${ }^{6}$ and is employed in this work to screen for aqueous stability.

\section{References}

(1) Benedek, R.; Thackeray, M.; Van De Walle, A. Pourbaix-like phase diagram for lithium manganese spinels in acid. Journal of Materials Chemistry 2010, 20, 369-374.

(2) Greeley, J.; Nørskov, J. K. Electrochemical dissolution of surface alloys in acids: Thermodynamic trends from first-principles calculations. Electrochimica Acta 2007, 52, 58295836.

(3) Persson, K. A.; Waldwick, B.; Lazic, P.; Ceder, G. Prediction of solid-aqueous equilibria: Scheme to combine first-principles calculations of solids with experimental aqueous states. Physical Review B 2012, 85, 235438.

(4) Singh, A. K.; Zhou, L.; Shinde, A.; Suram, S. K.; Montoya, J. H.; Winston, D.; Gregoire, J. M.; Persson, K. A. Electrochemical stability of metastable materials. Chemistry of Materials 2017, 29, 10159-10167.

(5) The decomposition energies in Ref. ${ }^{4}$ were normalized by the total number of non-oxygen atoms compared to the total number of atoms in the present study.

(6) Kirklin, S.; Saal, J. E.; Meredig, B.; Thompson, A.; Doak, J. W.; Aykol, M.; Rühl, S.;

Wolverton, C. The Open Quantum Materials Database (OQMD): assessing the accuracy of DFT formation energies. npj Computational Materials 2015, 1, 15010. 
Table S1: The convex hull stability $\left(E_{\text {hull }}\right)$, aqueous stability $\left(\Delta G_{a q}\right)$, GGA-DFT predicted bandgap $E_{\text {gap }}^{G G A}$, and decomposition products of filtered two-dimensional materials under acidic ORR and OER conditions. All materials from Materials Project are included in the determination of stabilities.

\begin{tabular}{|c|c|c|c|c|}
\hline Composition & $\begin{array}{c}E_{\text {hull }} \\
\text { (eV/atom) }\end{array}$ & $\begin{array}{c}\Delta G_{a q} \\
(\mathrm{eV} / \text { atom })\end{array}$ & $\begin{array}{l}E_{\text {gap }}^{G G A} \\
(\mathrm{eV})\end{array}$ & $\begin{array}{l}\text { Decomposition } \\
\text { products }\end{array}$ \\
\hline \multicolumn{5}{|c|}{ ORR, $\mathrm{pH}=1, \mathrm{U}=0.9 \mathrm{~V}$} \\
\hline $\mathrm{Si} 2 \mathrm{H} 2 \mathrm{O} 5$ & 0.07 & 0.01 & 5.70 & Si2H2O5(s) \\
\hline $\mathrm{Fe} 3 \mathrm{P} 2(\mathrm{HO}) 16$ & 0.10 & 0.04 & 2.33 & $\mathrm{FeHO} 2(\mathrm{~s})+\mathrm{FeP}(\mathrm{H} 2 \mathrm{O} 3) 2(\mathrm{~s})$ \\
\hline $\mathrm{P} 2 \mathrm{~Pb} 3 \mathrm{O} 8$ & 0.05 & 0.05 & 3.46 & $\mathrm{P} 2 \mathrm{~Pb} 3 \mathrm{O} 8(\mathrm{~s})$ \\
\hline $\mathrm{Ti} 3 \mathrm{H} 2 \mathrm{O} 7$ & 0.11 & 0.05 & 2.83 & Ti3H2O7(s) \\
\hline AgIO3 & 0.04 & 0.05 & 2.27 & $\mathrm{Ag}+(\mathrm{aq})+\mathrm{HIO} 3(\mathrm{~s})$ \\
\hline $\mathrm{BiOF}$ & 0.05 & 0.05 & 3.42 & $\mathrm{Bi} 2 \mathrm{O} 3(\mathrm{~s})+\mathrm{H} 3 \mathrm{OF}(\mathrm{s})$ \\
\hline $\mathrm{Sc}(\mathrm{IO} 3) 3$ & 0.03 & 0.05 & 3.98 & $\mathrm{HIO} 3(\mathrm{~s})+\mathrm{Sc}(\mathrm{HO}) 3(\mathrm{~s})$ \\
\hline $\mathrm{V} 2 \mathrm{~Pb} 3 \mathrm{O} 8$ & 0.04 & 0.06 & 3.17 & $\mathrm{~V} 3 \mathrm{HPb} 5 \mathrm{O} 13(\mathrm{~s})+\mathrm{VO} 2+(\mathrm{aq})$ \\
\hline $\mathrm{Zr}(\mathrm{IO} 3) 4$ & 0.05 & 0.06 & 3.30 & $\mathrm{HIO} 3(\mathrm{~s})+\mathrm{ZrOH}+++(\mathrm{aq})$ \\
\hline $\mathrm{PbWO} 4$ & 0.06 & 0.06 & 3.57 & PbWO4(s) \\
\hline FeHO2 & 0.10 & 0.06 & 1.91 & $\mathrm{FeHO} 2(\mathrm{~s})$ \\
\hline $\mathrm{H} 2 \mathrm{WO} 4$ & 0.13 & 0.06 & 1.49 & $\mathrm{H} 2 \mathrm{WO} 4(\mathrm{~s})$ \\
\hline V3(H3O5)2 & 0.09 & 0.07 & 1.23 & $\mathrm{VO} 2+(\mathrm{aq})$ \\
\hline $\mathrm{Bi} 2 \mathrm{O} 3$ & 0.07 & 0.07 & 3.02 & $\mathrm{Bi} 2 \mathrm{O} 3(\mathrm{~s})$ \\
\hline $\mathrm{Al}(\mathrm{HO}) 3$ & 0.10 & 0.07 & 4.38 & $\mathrm{Al}+++(\mathrm{aq})$ \\
\hline $\mathrm{Cd}(\mathrm{IO} 3) 2$ & 0.06 & 0.07 & 3.46 & $\mathrm{Cd}++(\mathrm{aq})+\mathrm{HIO} 3(\mathrm{~s})$ \\
\hline $\mathrm{CuH} 4(\mathrm{IO} 4) 2$ & 0.11 & 0.08 & 0.85 & $\mathrm{CuH} 4(\mathrm{IO} 4) 2(\mathrm{~s})$ \\
\hline $\mathrm{Al} 2 \mathrm{Si} 2 \mathrm{H} 4 \mathrm{O} 9$ & 0.05 & 0.08 & 4.48 & $\mathrm{Al}+++(\mathrm{aq})+\mathrm{Si} 2 \mathrm{H} 2 \mathrm{O} 5(\mathrm{~s})$ \\
\hline $\mathrm{GeO} 2$ & 0.11 & 0.11 & 3.54 & $\mathrm{GeO} 2(\mathrm{~s})$ \\
\hline GeBi2O5 & 0.15 & 0.15 & 2.13 & $\mathrm{Bi} 2 \mathrm{O} 3(\mathrm{~s})+\mathrm{Ge} 3(\mathrm{BiO} 3) 4(\mathrm{~s})$ \\
\hline Ge3Bi2O9 & 0.15 & 0.15 & 3.93 & $\mathrm{Ge} 3(\mathrm{BiO} 3) 4(\mathrm{~s})+\mathrm{GeO} 2(\mathrm{~s})$ \\
\hline \multicolumn{5}{|c|}{$\mathrm{OER}, \mathrm{pH}=1, \mathrm{U}=1.5 \mathrm{~V}$} \\
\hline Ti2O7 & 0.08 & -0.00 & 1.20 & $\mathrm{Ti} 2 \mathrm{O} 7(\mathrm{~s})$ \\
\hline $\mathrm{ZrO} 3$ & 0.10 & -0.00 & 3.23 & $\mathrm{ZrO} 3(\mathrm{~s})$ \\
\hline $\mathrm{NiO} 3$ & 0.06 & 0.00 & 0.00 & $\mathrm{NiO} 3(\mathrm{~s})$ \\
\hline $\mathrm{CuHO} 2$ & 0.05 & 0.01 & 0.74 & $\mathrm{CuHO} 2(\mathrm{~s})$ \\
\hline $\mathrm{AgO} 2 \mathrm{~F}$ & 0.02 & 0.02 & 0.00 & $\mathrm{AgO} 2 \mathrm{~F}(\mathrm{~s})$ \\
\hline $\mathrm{Pb}(\mathrm{BrO} 3) 2$ & 0.05 & 0.05 & 3.12 & $\mathrm{~Pb}(\mathrm{BrO} 3) 2(\mathrm{~s})$ \\
\hline AgHO2 & 0.10 & 0.06 & 0.00 & AgHO2(s) \\
\hline FeHO2 & 0.10 & 0.06 & 1.91 & $\mathrm{FeHO} 2(\mathrm{~s})$ \\
\hline $\mathrm{H} 2 \mathrm{WO} 4$ & 0.13 & 0.06 & 1.49 & $\mathrm{H} 2 \mathrm{WO} 4(\mathrm{~s})$ \\
\hline $\mathrm{Si} 2 \mathrm{H} 2 \mathrm{O} 5$ & 0.07 & 0.07 & 5.70 & Si6O13(s) \\
\hline TiO3 & 0.09 & 0.08 & 2.26 & $\mathrm{Ti} 2 \mathrm{O} 7(\mathrm{~s})$ \\
\hline $\mathrm{PbO} 2$ & 0.10 & 0.10 & 1.26 & $\mathrm{PbO} 2(\mathrm{~s})$ \\
\hline $\mathrm{GeO} 2$ & 0.11 & 0.11 & 3.54 & $\mathrm{GeO} 2(\mathrm{~s})$ \\
\hline $\mathrm{BiO} 2$ & 0.12 & 0.11 & 0.37 & $\mathrm{BiO} 2(\mathrm{~s})$ \\
\hline
\end{tabular}


Table S2: The convex hull stability $\left(E_{\text {hull }}\right)$, aqueous stability $\left(\Delta G_{a q}\right)$, GGA-DFT predicted bandgap $E_{\text {gap }}^{G G A}$, and decomposition products of filtered two-dimensional materials under basic ORR and OER conditions. All materials from Materials Project are included in the determination of stabilities.

\begin{tabular}{|c|c|c|c|c|}
\hline Composition & $\begin{array}{c}E_{\text {hull }} \\
(\mathrm{eV} / \text { atom })\end{array}$ & $\begin{array}{c}\Delta G_{a q} \\
(\mathrm{eV} / \text { atom })\end{array}$ & $\begin{array}{c}E_{g a p}^{G G A} \\
(\mathrm{eV})\end{array}$ & $\begin{array}{l}\text { Decomposition } \\
\text { products }\end{array}$ \\
\hline \multicolumn{5}{|c|}{ ORR, $\mathrm{pH}=13, \mathrm{U}=0.9 \mathrm{~V}$} \\
\hline $\mathrm{ZrO} 3$ & 0.10 & -0.00 & 3.23 & $\mathrm{ZrO} 3(\mathrm{~s})$ \\
\hline $\mathrm{NiO} 3$ & 0.06 & 0.00 & 0.00 & $\mathrm{NiO} 3(\mathrm{~s})$ \\
\hline Ti2O7 & 0.08 & 0.00 & 1.20 & $\mathrm{Ti} 2 \mathrm{O} 7(\mathrm{~s})$ \\
\hline $\mathrm{CuHO} 2$ & 0.05 & 0.01 & 0.74 & $\mathrm{CuHO} 2(\mathrm{~s})$ \\
\hline $\mathrm{AgO} 2 \mathrm{~F}$ & 0.02 & 0.02 & 0.00 & $\mathrm{AgO} 2 \mathrm{~F}(\mathrm{~s})$ \\
\hline $\mathrm{Ni}(\mathrm{HO}) 2$ & 0.06 & 0.03 & 2.22 & $\mathrm{NiHO} 2(\mathrm{~s})$ \\
\hline $\mathrm{Si} 2 \mathrm{H} 2 \mathrm{O} 5$ & 0.07 & 0.03 & 5.70 & $\mathrm{HSi}(\mathrm{OH}) 6-(\mathrm{aq})$ \\
\hline $\mathrm{Al}(\mathrm{HO}) 3$ & 0.10 & 0.05 & 4.38 & $\mathrm{Al}(\mathrm{HO}) 3(\mathrm{~s})$ \\
\hline $\mathrm{Pb}(\mathrm{BrO} 3) 2$ & 0.05 & 0.05 & 3.12 & $\mathrm{~Pb}(\mathrm{BrO} 3) 2(\mathrm{~s})$ \\
\hline $\mathrm{AgHO} 2$ & 0.10 & 0.06 & 0.00 & $\mathrm{AgHO} 2(\mathrm{~s})$ \\
\hline $\mathrm{Co} 3 \mathrm{NiO} 8$ & 0.11 & 0.06 & 1.30 & $\mathrm{CoO} 2(\mathrm{~s})+\mathrm{NiHO} 2(\mathrm{~s})$ \\
\hline Al2Si2H4O9 & 0.05 & 0.07 & 4.48 & $\mathrm{Al}(\mathrm{HO}) 3(\mathrm{~s})+\mathrm{HSi}(\mathrm{OH}) 6-(\mathrm{aq})$ \\
\hline FeHO2 & 0.10 & 0.07 & 1.91 & $\mathrm{FeO} 4-(\mathrm{aq})$ \\
\hline $\mathrm{Co} 2 \mathrm{NiO} 6$ & 0.12 & 0.08 & 1.28 & $\mathrm{CoO} 2(\mathrm{~s})+\mathrm{NiHO} 2(\mathrm{~s})$ \\
\hline $\mathrm{Zn}(\mathrm{HO}) 2$ & 0.14 & 0.10 & 2.34 & $\mathrm{Zn}(\mathrm{HO}) 2(\mathrm{~s})$ \\
\hline $\mathrm{PbO} 2$ & 0.10 & 0.10 & 1.26 & $\mathrm{PbO} 2(\mathrm{~s})$ \\
\hline $\mathrm{BiO} 2$ & 0.12 & 0.11 & 0.37 & $\mathrm{BiO} 2(\mathrm{~s})$ \\
\hline \multicolumn{5}{|c|}{ OER, $\mathrm{pH}=13, \mathrm{U}=1.5 \mathrm{~V}$} \\
\hline $\mathrm{Bi}(\mathrm{MoO} 5) 2$ & 0.06 & -0.00 & 1.96 & $\mathrm{Bi}(\mathrm{MoO} 5) 2(\mathrm{~s})$ \\
\hline $\mathrm{ZrO} 3$ & 0.10 & -0.00 & 3.23 & $\mathrm{ZrO} 3(\mathrm{~s})$ \\
\hline $\operatorname{Mg} 3(\mathrm{PO} 8) 2$ & 0.14 & -0.00 & 0.00 & Mg3(PO8)2(s) \\
\hline $\mathrm{NiO} 3$ & 0.06 & 0.00 & 0.00 & $\mathrm{NiO} 3(\mathrm{~s})$ \\
\hline $\mathrm{Co} 2 \mathrm{NiO} 6$ & 0.12 & 0.00 & 1.28 & $\mathrm{Co} 2 \mathrm{NiO} 6(\mathrm{~s})$ \\
\hline $\mathrm{Co} 3 \mathrm{NiO} 8$ & 0.11 & 0.00 & 1.30 & Co3NiO8(s) \\
\hline $\mathrm{Ti} 2 \mathrm{O} 7$ & 0.08 & 0.00 & 1.20 & $\mathrm{Ti} 2 \mathrm{O} 7(\mathrm{~s})$ \\
\hline $\mathrm{CuO} 2 \mathrm{~F}$ & 0.01 & 0.01 & 0.84 & $\mathrm{CuO} 2 \mathrm{~F}(\mathrm{~s})$ \\
\hline $\mathrm{CuHO} 2$ & 0.05 & 0.01 & 0.74 & $\mathrm{CuHO} 2(\mathrm{~s})$ \\
\hline $\mathrm{AgO} 2 \mathrm{~F}$ & 0.02 & 0.02 & 0.00 & $\mathrm{AgO} 2 \mathrm{~F}(\mathrm{~s})$ \\
\hline $\mathrm{Al}(\mathrm{HO}) 3$ & 0.10 & 0.05 & 4.38 & $\mathrm{Al}(\mathrm{HO}) 3(\mathrm{~s})$ \\
\hline AgHO2 & 0.10 & 0.06 & 0.00 & AgHO2(s) \\
\hline $\mathrm{Zn}(\mathrm{HO}) 2$ & 0.14 & 0.10 & 2.34 & $\mathrm{Zn}(\mathrm{HO}) 2(\mathrm{~s})$ \\
\hline $\mathrm{PbO} 2$ & 0.10 & 0.10 & 1.26 & $\mathrm{PbO} 2(\mathrm{~s})$ \\
\hline $\mathrm{BiO} 2$ & 0.12 & 0.11 & 0.37 & $\mathrm{BiO} 2(\mathrm{~s})$ \\
\hline
\end{tabular}

